\title{
Optimal Signal Design For Estimation Of Correlated MIMO Channels
}

\author{
Jayesh H. Kotecha and Akbar M. Sayeed \\ Dept. of ECE, University of Wisconsin at Madison, Madison, WI 53706. \\ Email: jkotecha@ece.wisc.edu,akbar@engr.wisc.edu.
}

\begin{abstract}
We address signal design for optimal estimation of correlated multi-input multi-output (MIMO) channels using pilot signals, assuming knowledge of the second-order channel statistics at the transmitter. Assuming a block fading channel model and minimum mean square error (MMSE) estimation at the receiver, we design the transmitted signal to optimize two criteria: MMSE and the conditional mutual information between the MIMO channel and the received signal. Our analysis is based on the recently proposed virtual channel representation, however, it is generalized to other known channel models like the one in [1]. We show that optimal signaling is in a block form, where the block length depends on the signal to noise ratio (SNR) as well as the channel correlation matrix. The block signal corresponds to transmitting beams in successive time intervals along fixed virtual transmit angles, whose powers are determined by (non-identical) water filling arguments based on the optimization criteria. Our analysis effectively provides characterization of the dominant subspaces of the channel as a function of the SNR and the scattering environment, which is critical to achieving capacity and space-time code design. In particular, at low SNR the block length reduces to one and all the power is transmitted on the beam corresponding to the strongest transmit angle, while at high SNR the block length has a maximum length equal to the number of active virtual transmit angles and the power is assigned equally to all active transmit angles. Consequently, from a channel estimation viewpoint, a faster fading rate can be tolerated at low SNRs relative to higher SNRs.
\end{abstract}

\section{INTRODUCTION}

Multi-antennae communication systems are gaining prominence due to the higher capacity and reliability they can afford [2],[3]. Perfect knowledge of channel state is often assumed in the analysis of such systems. However, in practice the channel has to be estimated, typically using pilot symbols. In a rich scattering environment, the assumption of i.i.d. channels is valid and multi-input multi-output (MIMO) channel estimation can be straightforwardly done using, for example, least squares or minimum mean square error (MMSE) techniques [4]. However, this idealized assumption does not necessarily hold and hence a study of correlated channels is of interest. In this work, we investigate transmit signal design for optimal estimation of correlated MIMO Rayleigh fading channels, assuming that the receiver and the transmitter ${ }^{1}$ have knowledge of the second order statistics of the MIMO channel ${ }^{2}$. It is assumed that the receiver uses an MMSE channel estimator. The covariance feedback information is exploited by the transmitter to design the transmit signal to minimize the channel estimation error at the receiver.

This research is supported in part by NSF Grant Nos. CCR-9875805 and CCR-0113385 and ONR Grant No. N00014-01-1-0825.

${ }^{1}$ This is often called covariance feedback.

${ }^{2}$ The assumption is reasonable, since the second order statistics are much less dynamic than the channel itself. Thus, they can be estimated more reliably and need to be updated less frequently.
We assume a block flat fading model, where the channel is constant over a block of transmitted symbols, but changes independently from block to block. We design the transmit signal to optimize one of two criteria: minimization of the MMSE at the receiver or maximization of the conditional mutual information between the channel and the received signal.

A MIMO channel with $P$ transmit and $Q$ receive antennae has a maximum of $P Q$ unknowns to be estimated. However, correlated MIMO channels possess fewer degrees of freedom and hence fewer than $P Q$ parameters, corresponding to the degrees of freedom, need to be estimated. Our analysis is based on the recently proposed virtual MIMO channel representation [5] for uniform linear arrays (ULA) at both the transmitter and receiver. The virtual model characterizes the channel in the spatial domain by beamforming in the direction of fixed virtual angles determined by the spatial resolution of the antenna arrays, which is analogous to representing the channel in beamspace or wavenumber domain. The non-vanishing and approximately uncorrelated elements of the virtual channel matrix represent the degrees of freedom in the channel. These degrees of freedom are governed by the scattering geometry, the antennae spacings and the number of antennas. Channel estimation can now be viewed as the identification of this scattering geometry in the virtual domain and the gains it imposes on the input and output antennae. The analysis can be generalized to other channel models, an example is the channel where the correlation in the transmit and receive arrays induce correlation in the rows and columns of the channel matrix [1].

We show that the optimal transmit signal is a block signal consisting of beams transmitted in succession along the active virtual transmit angles, corresponding to directions in which scatterers are present. We assume that, while the total transmit power in a block is constant, the transmit power during each transmission may be different. The power transmitted along the beams is determined by water filling arguments resulting from the two criteria. Power is possibly assigned to a beam only if the second-order statistics indicate the presence of significant scattering in that direction. However, the power assigned to the transmit beams depends on the signal to noise ratio (SNR) as well. Equivalently, the scattering environment is scanned along the virtual transmit angles one by one to determine the presence of scattering clusters, by measuring the signals along the virtual receive angles for each transmitted beam. Hence, the optimal signal design suggests that, for a given SNR, only those columns of the virtual channel matrix are estimated that are deemed dominant by the water filling criteria. This effectively characterizes the dominant subspaces of the channel as a function of the SNR and the scattering environment and is an important outcome of the analysis which can be further used in 
other situations. For instance, space-time code design can now be done as a function of SNR, where only the dominant elements of the channel matrix are considered in the design. Notably, capacity results in the case of imperfect channel state information indicate that identification of such dominant degrees of freedom is critical to achieving capacity [6].

Section II introduces the MIMO channel and briefly describes the virtual representation. Section III discusses MMSE estimation of the MIMO channel and motivates criteria for optimum signal design which are presented in Section IV. Section $\mathrm{V}$ presents numerical simulation results. ${ }^{3}$

\section{MIMO CHANNEL MODEL}

Consider a narrowband frequency non-selective MIMO channel with $P$ transmit and $\mathrm{Q}$ receive antennae. With $k$ indicating discrete time, if $\mathbf{s}(k)$ is the transmit vector of dimension $P$, then the $Q$ dimensional received signal $\mathbf{x}(k)$ can be written as

$$
\mathbf{x}(k)=\mathbf{H}(k) \mathbf{s}(k)+\mathbf{n}(k)
$$

where $\mathbf{H}(k)$ is the $Q \times P$ channel gain matrix. $\mathbf{n}(k)$ is the $Q$ dimensional zero mean, complex white Gaussian noise vector, with covariance matrix $\sigma^{2} \mathbf{I}_{Q}$. The channel gain between the $n-t h$ receive and $m-t h$ transmit antenna is denoted by $\mathbf{H}[m, n]$.

In [5], the virtual channel representation is proposed where the transmit and receive antennae are uniform linear arrays (ULA). If $d_{T}$ and $d_{R}$ are the transmit and receive array spacings, then $\mathbf{H}$ can be related to the physical propagation environment via the array steering and response vectors $\mathbf{a}_{T}\left(\theta_{T}\right)=\frac{1}{\sqrt{P}}\left[1, \exp \left(-j 2 \pi \theta_{T}\right), \ldots, \exp \left(-j 2 \pi(P-1) \theta_{T}\right)\right]^{T}$, $\mathbf{a}_{R}\left(\theta_{R}\right)=\frac{1}{\sqrt{Q}}\left[1, \exp \left(-j 2 \pi \theta_{R}\right), \ldots, \exp \left(-j 2 \pi(Q-1) \theta_{R}\right)\right]^{T}$ where the $\theta$ is the delay between the signals received at adjacent elements in the array due to a point source at angle $\phi$ (relative to a horizontal axis). If $\lambda$ is the wavelength of propagation, then $\theta=\frac{d}{\lambda} \sin \phi$. We will interpret $\theta$ as a normalized angle. The linear virtual channel representation in [5] exploits the finite dimensionality of the spatial signal space arising from finite number of array elements and finite array apertures. Without loss of generality, assume $P$ and $Q$ to be odd and define $\tilde{Q}=(Q-1) / 2$ and $\tilde{P}=(P-1) / 2$. The virtual channel representation is given by

$$
\mathbf{H}=\sum_{q=-\tilde{Q}}^{\tilde{Q}} \sum_{p=-\tilde{P}}^{\tilde{P}} \mathbf{H}_{V}[p, q] \mathbf{a}_{R}\left(\tilde{\theta}_{R, q}\right) \mathbf{a}_{T}^{H}\left(\tilde{\theta}_{T, p}\right)=\tilde{\mathbf{A}}_{R} \mathbf{H}_{V} \tilde{\mathbf{A}}_{T}^{H}
$$

where $\tilde{\mathbf{A}}_{R}=\left[\mathbf{a}_{R}\left(\tilde{\theta}_{R,-\tilde{Q}}\right), \ldots, \mathbf{a}_{R}\left(\tilde{\theta}_{R,+\tilde{Q}}\right)\right](Q \times Q)$ and $\tilde{\mathbf{A}}_{T}=$ $\left[\mathbf{a}_{T}\left(\tilde{\theta}_{T,-\tilde{Q}}\right), \ldots, \mathbf{a}_{T}\left(\tilde{\theta}_{T,+\tilde{Q}}\right)\right](P \times P)$ are defined by the fixed virtual angles $\tilde{\theta}_{R, q}$ and $\tilde{\theta}_{T, p}$ and are full rank. We assume that the spatial virtual angles are uniformly spaced [5] and hence $\tilde{\mathbf{A}}_{T}$ and $\tilde{\mathbf{A}}_{R}$ are discrete Fourier transform matrices (and hence

\footnotetext{
${ }^{3}$ Notation: For an integer $Q, \mathbf{I}_{Q}$ is a $Q \times Q$ identity matrix. If $\mathbf{X}$ is a $Q \times K$ matrix, $\operatorname{vec}(\mathbf{X})$ denotes the $Q K \times 1$ vector obtained by stacking columns of $\mathbf{X}$. $\otimes$ denotes the Kronecker product [7]. The inverse and pseudo-inverse of $\mathbf{X}$ are denoted by $\mathbf{X}^{-1}$ and $\mathbf{X}^{\dagger} \cdot \operatorname{tr}(\mathbf{X})$ denotes the trace of the square matrix X. $\operatorname{diag}\left(\left[a_{1}, \ldots, a_{Q}\right]\right)$ is a $Q \times Q$ diagonal matrix with diagonal elements $a_{1}, \ldots, a_{Q} . E(\cdot)$ denotes the expectation operator. $(x)^{+}=\max (0, x)$.
}

unitary). Note that the virtual model is linear in the gains and spatial angles, since these angles are fixed a priori. Note that we can write $\mathbf{h}=\operatorname{vec}(\mathbf{H})=\left(\tilde{\mathbf{A}}_{T}^{*} \otimes \tilde{\mathbf{A}}_{R}\right) \mathbf{h}_{V}$. The resulting channel correlation has a Kronecker structure given by

$$
\mathbf{R}=E\left(\mathbf{h} \mathbf{h}^{H}\right)=\left(\tilde{\mathbf{A}}_{T}^{*} \otimes \tilde{\mathbf{A}}_{R}\right) \mathbf{R}_{V}\left(\tilde{\mathbf{A}}_{T}^{*} \otimes \tilde{\mathbf{A}}_{R}\right)^{H} .
$$

An important consequence of the virtual modelling is that, the elements of $\mathbf{H}_{V}$ are approximately uncorrelated and hence $\mathbf{R}_{V}$ is approximately diagonal regardless of the correlation structure of $\mathbf{R}$ [5]. The structure obtained by the virtual model allows simplification in signal design and provides interesting interpretations as shall be seen.

The techniques developed in this paper can be straightforwardly generalized to channels where the channel matrix can be expressed as

$$
\mathbf{H}=\mathbf{U}_{R} \mathbf{H}_{V} \mathbf{U}_{T}^{H}
$$

where $\mathbf{U}_{T}$ and $\mathbf{U}_{R}$ are the transmit and receive unitary matrices and the elements of $\mathbf{H}_{V}$ are uncorrelated but not necessarily identically distributed. The resulting channel correlation has a Kronecker structure similar to (2). Such channel models may arise as a consequence of the array geometry as was seen above in the case of ULAs. Another example is the channel model, where it is assumed that the transmitter and receiver antennae arrays have correlated elements [1]. The channel matrix can be written as

$$
\mathbf{H}=\boldsymbol{\Sigma}_{R}^{1 / 2} \mathbf{H}_{w} \boldsymbol{\Sigma}_{T}^{1 / 2}=\mathbf{U}_{R} \mathbf{H}_{V} \mathbf{U}_{T}^{H}
$$

where the elements of $\mathbf{H}_{w}$ are i.i.d. The matrices $\boldsymbol{\Sigma}_{T}$ and $\boldsymbol{\Sigma}_{T}$ are the transmit and receive array correlation matrices with eigen value decompositions (EVD) $\mathbf{U}_{T} \Lambda_{T} \mathbf{U}_{T}^{H}$ and $\mathbf{U}_{R} \Lambda_{R} \mathbf{U}_{R}^{H}$ respectively. The elements of $\mathbf{H}_{V}$ are uncorrelated with diagonal covariance matrix given by $\mathbf{R}_{V}=\Lambda_{T} \otimes \Lambda_{R}$ [8].

Since $\mathbf{H}$ and $\mathbf{H}_{V}$ are unitarily equivalent, estimation of the MIMO channel can be equivalently performed by obtaining estimates of $\mathbf{H}_{V}$. From (1) and (3), we can write the received signal as

$$
\mathbf{x}(k)=\tilde{\mathbf{A}}_{R} \mathbf{H}_{V}(k) \tilde{\mathbf{A}}_{T}^{H} \mathbf{s}(k)+\mathbf{n}(k) .
$$

In the eigen or virtual domain,

$$
\mathbf{x}_{V}(k)=\mathbf{H}_{V}(k) \mathbf{s}_{V}(k)+\mathbf{n}_{V}(k)
$$

where $\mathbf{x}_{V}=\tilde{\mathbf{A}}_{R}^{H} \mathbf{x}$ and $\mathbf{s}_{V}=\tilde{\mathbf{A}}_{T}^{H} \mathbf{s}$ are the projections of the received and transmitted signals onto the fixed receive and transmit response vectors respectively. Equation (6) provides an interesting interpretation of transmission in the virtual domain. Each element of $\mathbf{x}_{V}\left(\mathbf{s}_{V}\right)$ corresponds to a signal received (transmitted) from (to) the fixed virtual angles $\tilde{\theta}_{T, p}\left(\tilde{\theta}_{R, q}\right)$ and the corresponding element in $\mathbf{H}_{V}$ indicates the coupling gain between these angles [5]. Note that since $\tilde{\mathbf{A}}_{R}$ is unitary, $\mathbf{n}_{V}=$ $\tilde{\mathbf{A}}_{R} \mathbf{n}$ is zero mean, white Gaussian with covariance $\sigma^{2} \mathbf{I}_{q}$.

In the following development, we assume the MIMO channel to be block fading, i.e. $\mathbf{H}(k)=\mathbf{H}$ for $k=1, \ldots, K$ and the channel is independent between different blocks of $K$ symbols. Assuming that training symbols $\mathbf{s}(k), k=1, \ldots, K$ are sent in a block mode and denoting $\mathbf{S}=[\mathbf{s}(1), \ldots, \mathbf{s}(K)]$, the block fading model is given by

$$
\mathbf{X}_{V}=\mathbf{H}_{V} \mathbf{S}_{V}+\mathbf{N}_{V}
$$


where $\mathbf{X}_{V}=\left[\mathbf{x}_{V}(1), \ldots, \mathbf{x}_{V}(K)\right], \mathbf{S}_{V}=\left[\mathbf{s}_{V}(1), \ldots, \mathbf{s}_{V}(K)\right]$ and $\mathbf{N}_{V}=\left[\mathbf{n}_{V}(1), \ldots, \mathbf{n}_{V}(K)\right]$. Stacking the columns of $\mathbf{X}_{V}$, we obtain

$\tilde{\mathbf{x}}_{V}=\operatorname{vec}\left(\mathbf{X}_{V}\right)=\left(\mathbf{S}_{V}^{T} \otimes \mathbf{I}_{Q}\right) \operatorname{vec}\left(\mathbf{H}_{V}\right)+\operatorname{vec}\left(\mathbf{N}_{V}\right)=\tilde{\mathbf{S}}_{V} \mathbf{h}_{V}+\tilde{\mathbf{n}}_{V}$

where we denote $\tilde{\mathbf{S}}_{V}=\left(\mathbf{S}_{V}^{T} \otimes \mathbf{I}_{Q}\right)$. Using (7), we proceed with the estimation of $\mathbf{h}_{V}$, which is a $P Q$ vector. Clearly, since the maximum number of unknowns in $\mathbf{h}_{V}{ }^{4}$ is $P Q$, we need to transmit a block of $K \leq P$ symbols [9]. Hence, we need the quasi-static channel to be constant for only $K \leq P$ time periods.

\section{MMSE AND MAP ESTIMATION}

The model (7) is linear in $\mathbf{h}_{V}$ and Gaussian. Hence, it can be shown that the linear MMSE estimate, the MMSE estimate and the MAP estimate are identical. In this paper, we assume that the covariance matrix $\mathbf{R}_{V}=E\left(\mathbf{h}_{V} \mathbf{h}_{V}^{H}\right.$ ) (or equivalently $\mathbf{R}=$ $\left.E\left(\mathbf{h} \mathbf{h}^{H}\right)\right)$ is known. The linear MMSE estimator minimizes the error MSE $=E\left[\left\|\mathbf{h}_{V}-\hat{\mathbf{h}}_{V}\right\|^{2}\right]$. The resulting linear estimate is

$$
\hat{\mathbf{h}}_{V}=\mathbf{G}_{o p t} \tilde{\mathbf{x}}_{V}
$$

where $\mathbf{G}_{o p t}$ is a $P Q \times P Q$ matrix given by

$\mathbf{G}_{o p t}=\arg \min _{\mathbf{G}} E\left[\left\|\mathbf{h}_{V}-\mathbf{G} \tilde{\mathbf{x}}_{V}\right\|^{2}\right]=\mathbf{R}_{V} \tilde{\mathbf{S}}_{V}^{H}\left(\tilde{\mathbf{S}}_{V} \mathbf{R}_{V} \tilde{\mathbf{S}}_{V}^{H}+\sigma^{2} \mathbf{I}\right)^{-1}$.

Using the orthogonality principle, the error covariance matrix and the minimum MSE are

$$
\begin{aligned}
\mathbf{C}_{e} & =\mathbf{R}_{V}-\mathbf{R}_{V} \tilde{\mathbf{S}}_{V}^{H}\left(\tilde{\mathbf{S}}_{V} \mathbf{R}_{V} \tilde{\mathbf{S}}_{V}^{H}+\sigma^{2} \mathbf{I}\right)^{-1} \tilde{\mathbf{S}}_{V} \mathbf{R}_{V} \\
& =\left(\mathbf{R}_{V}^{\dagger}+\frac{1}{\sigma^{2}} \tilde{\mathbf{S}}_{V}^{H} \tilde{\mathbf{S}}_{V}\right)^{-1}, \\
\mathrm{MMSE} & =\operatorname{tr}\left(\mathbf{C}_{e}\left(\tilde{\mathbf{S}}_{V}\right)\right) .
\end{aligned}
$$

respectively. Moreover, if $\mathbf{h}_{V}$ is assumed to be zero mean Gaussian with covariance $\mathbf{R}_{V}$, then the linear estimate in (8) is also the maximum a posteriori estimate. The conditional mutual information (CMI) between the received signal and the channel $\mathbf{h}_{V}$ is given by

$$
\operatorname{CMI}\left(\tilde{\mathbf{S}}_{V}\right)=\log \operatorname{det}\left(\mathbf{I}+\frac{1}{\sigma^{2}} \tilde{\mathbf{S}}_{V} \mathbf{R}_{V} \tilde{\mathbf{S}}_{V}^{H}\right)
$$

Note that, $\mathbf{C}_{e}$ is a function $\mathbf{S}_{V}$. In the next section, we consider optimal signal design of the transmit block $\mathbf{S}_{V}$ to satisfy two optimization criteria.

\section{Optimum Signal Design}

We consider the design of the optimum transmit block signal $\tilde{\mathbf{S}}_{V}$ (or equivalently $\mathbf{S}_{V}$ ) with respect to two criteria: $\min -$ imization of the MMSE (11) and maximization of the mutual information (12) between the channel and received signal conditioned on the transmitted block signal. We state the two optimization problems as follows:

$$
\min _{\tilde{\mathbf{S}}_{V}} \operatorname{tr}\left(\mathbf{R}_{V}^{\dagger}+\frac{1}{\sigma^{2}} \tilde{\mathbf{S}}_{V}^{H} \tilde{\mathbf{S}}_{V}\right)^{-1} \text { s.t. } \operatorname{tr}\left(\tilde{\mathbf{S}}_{V}^{H} \tilde{\mathbf{S}}_{V}\right) \leq P \beta,
$$

\footnotetext{
${ }^{4}$ The number of unknowns in $\mathbf{h}_{V}$ would be smaller in correlated channels. If the prior variance of a given element of $\mathbf{h}_{V}$ is zero, then it implies that the element is itself zero.
}

$\max _{\tilde{\mathbf{S}}_{V}} \log \operatorname{det}\left(\mathbf{I}+\frac{1}{\sigma^{2}} \tilde{\mathbf{S}}_{V} \mathbf{R}_{V} \tilde{\mathbf{S}}_{V}^{H}\right)$ s.t. $\operatorname{tr}\left(\tilde{\mathbf{S}}_{V}^{H} \tilde{\mathbf{S}}_{V}\right) \leq P \beta$,

where $\beta$ is the total transmitted power and $P$ is the number of transmit antennae. Note that the constraint $\operatorname{tr}\left(\tilde{\mathbf{S}}_{V}^{H} \tilde{\mathbf{S}}_{V}\right) \leq$ $P \beta$ is equivalent to the finite power constraint $\operatorname{tr}\left(\mathbf{S}_{V}^{H} \mathbf{S}_{V}\right)=$ $\operatorname{tr}\left(\mathbf{S}^{H} \mathbf{S}\right) \leq \beta$. We develop the signal design using the SVD of the transmitted block matrix. Denote the SVDs of $\mathbf{S}_{V}^{T}=$ $\mathbf{U}_{S} \Lambda_{S} \mathbf{V}_{S}^{H}$ and $\tilde{\mathbf{S}}_{V}=\mathbf{U}_{\tilde{S}} \Lambda_{\tilde{S}} \mathbf{V}_{\tilde{S}}^{H}$, where $\mathbf{U}_{S}, \mathbf{V}_{S}, \mathbf{U}_{\tilde{S}}$ and $\mathbf{V}_{\tilde{S}}$ are unitary matrices and $\Lambda_{S}$ and $\Lambda_{\tilde{S}}$ are diagonal matrices. Since $\tilde{\mathbf{S}}_{V}=\left(\mathbf{S}_{V}^{T} \otimes \mathbf{I}_{Q}\right)$, it follows that $\mathbf{U}_{\tilde{S}}=\mathbf{U}_{S} \otimes \mathbf{I}_{Q}$, $\Lambda_{\tilde{S}}=\Lambda_{S} \otimes \mathbf{I}_{Q}$ and $\mathbf{V}_{\tilde{S}}=\mathbf{V}_{S} \otimes \mathbf{I}_{Q}$. The following theorem states our main result, for a proof see [8].

Theorem 1: Consider the constrained optimization problems in (13) and (14) respectively. The globally optimal solution has a structure given by

$$
\tilde{\mathbf{S}}_{V, o p t}=\tilde{\Lambda}_{o p t} \mathbf{V}_{\tilde{S}}
$$

where $\tilde{\Lambda}_{\text {opt }} \in \mathbb{R}^{P Q \times P Q}$. The optimal $\mathbf{V}_{\tilde{S}}$ is a matrix of the eigenvectors of $\mathbf{R}_{V}$, i.e $\mathbf{V}_{\tilde{S}}=\mathbf{I}$ and $\tilde{\Lambda}_{\text {opt }}$ is the solution to

$$
\begin{gathered}
\tilde{\Lambda}_{\text {opt }}=\tilde{\Lambda}_{M}=\arg \min _{\Lambda_{\tilde{S}}} \operatorname{tr}\left(\mathbf{R}_{V}^{\dagger}+\frac{1}{\sigma^{2}} \Lambda_{\tilde{S}}^{H} \Lambda_{\tilde{S}}\right)^{-1} \\
\text { s.t. } \operatorname{tr}\left(\Lambda_{\tilde{S}}^{H} \Lambda_{\tilde{S}}\right) \leq P \beta
\end{gathered}
$$

$$
\text { and } \begin{aligned}
\tilde{\Lambda}_{o p t}=\tilde{\Lambda}_{C}= & \arg \max _{\Lambda_{\tilde{S}}} \log \operatorname{det}\left(\mathbf{I}+\Lambda_{\tilde{S}} \mathbf{R}_{V} \Lambda_{\tilde{S}}^{H}\right) \\
& \text { s.t. } \operatorname{tr}\left(\Lambda_{\tilde{S}}^{H} \Lambda_{\tilde{S}}\right) \leq P \beta
\end{aligned}
$$

respectively.

Since $\tilde{\mathbf{S}}_{V}=\left(\mathbf{S}_{V}^{T} \otimes \mathbf{I}_{Q}\right)$, from Theorem 1 the optimal transmit signal is $\mathbf{S}_{V}=\Lambda_{\text {opt }}$ where $\Lambda_{\text {opt }}$ is the solution to

$$
\begin{aligned}
& \arg \min _{\Lambda_{S}} \sum_{i=1}^{P} \sum_{j=1}^{Q}\left(\frac{\sigma^{2} \mathbf{R}_{V}[(i-1) Q+j,(i-1) Q+j]}{\sigma^{2}+\mathbf{R}_{V}[(i-1) Q+j,(i-1) Q+j] \beta_{i}}\right) \\
& \arg \max _{\Lambda_{S}} \sum_{i=1}^{P} \sum_{j=1}^{Q} \log \left(1+\frac{\mathbf{R}_{V}[(i-1) Q+j,(i-1) Q+j] \beta_{i}}{\sigma^{2}}\right)
\end{aligned}
$$

subject to the constraint $\sum_{i=1}^{P} \beta_{i} \leq \beta, \beta_{i}=\left|\Lambda_{\text {opt }}[i, i]\right|^{2}$, for the MMSE and CMI criteria respectively. Thus, the optimal transmit signal is a block diagonal signal (in the virtual domain). The optimal signal structure specifies that during the $P$ block transmission, at each time instant $i \in 1, \ldots, P$, the signal is transmitted along the $i$-th transmit eigen vector with the powers specified by $\beta_{i}$. Due to the diagonal structure of $\mathbf{S}_{V}, \mathbf{G}_{o p t}$ (9) and $\mathbf{C}_{e}$ (10) become diagonal, which enables independent processing at the receiver. The channel estimate is given by

$$
\begin{aligned}
& \hat{\mathbf{h}}_{V}[(i-1) Q+j]= \\
& \left(\frac{\mathbf{R}_{V}[(i-1) Q+j,(i-1) Q+j] \Lambda_{\text {opt }}[i, i]}{\sigma^{2}+\mathbf{R}_{V}[(i-1) Q+j,(i-1) Q+j]\left|\Lambda_{o p t}[i, i]\right|^{2}}\right) \cdot \mathbf{x}_{V}[(i-1) Q+j],
\end{aligned}
$$

for $j=1, \ldots, Q ; i=1, \ldots, P$. From this equation, note that the $i$-th transmission allows us to estimate the $Q$ elements in 


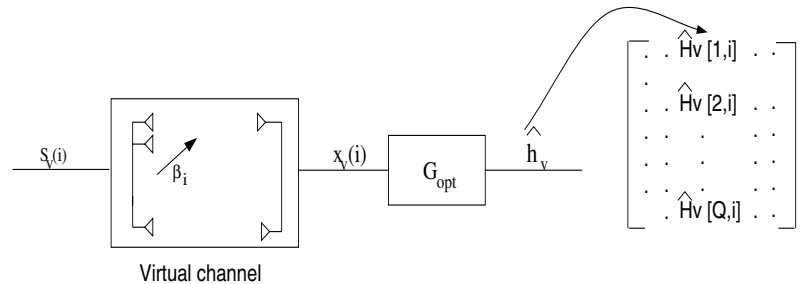

Fig. 1. Physical interpretation of channel estimation - 'Scanning' the scattering environment in the virtual domain. The i-th beam is used to estimate the i-th column of $\mathbf{H}_{V}$.

the $i-t h$ column of $\mathbf{H}_{V}$, i.e $\left(\mathbf{h}_{V}[(i-1) Q+1], \ldots, \mathbf{h}_{V}[(i-\right.$ $1) Q+Q] \quad$ ). During the block transmission, the scattering environment is scanned sequentially to estimate each column of $\mathbf{H}_{V}$. This scanning of the virtual domain is illustrated in Figure 1.

The constrained nonlinear optimizations in (18) and (19) are the so called 'water-filling' problems and can be solved using Langrange multipliers and using the Kuhn-Tucker conditions to verify that the solutions are non-negative. However, for the general case of $P$ transmit and $Q$ receive antennae, we have not been able to find a closed form solution and hence it has to be obtained numerically. In the following, we obtain approximate closed form solutions in the low SNR and high SNR regions to obtain some insight. Closed form solutions exist for the special cases of a MISO channel and the transmit and receive correlated channel (4) where either $\Sigma_{T}$ or $\Sigma_{R}$ is equal to $\sigma^{2} \mathbf{I}$, (both criteria give identical power assignment in these cases) for details see [8].

In the following discussion, the channel coefficient $\mathbf{H}_{V}[j, i]$ is defined as active if $E\left(\left|\mathbf{H}_{V}[j, i]\right|^{2}\right)>\epsilon$ for some prescribed $\epsilon>0$. Thus the set of active elements can be determined a priori by examining $\mathbf{R}_{V}$. Also, a column of the channel matrix $\mathbf{H}_{V}$ is defined as active column if it contains at least one active element. Let $A \subset\{1, \ldots, P\}$ be the set of active columns. Then for any $i \in A, Q_{i}$ is the number of active elements in the $i$-th column. We define the transmitted signal to noise ratio (TSNR) as the ratio of the transmitted signal power to the noise power $\frac{\beta}{\sigma^{2}}$ and the per virtual angle pair received signal to noise ratio (RSNR) as the ratio of the received signal power to the noise power $\operatorname{RSNR}(i, j)=\frac{\mathbf{R}_{V}[(i-1) Q+j,(i-1) Q+j] \beta_{i}}{\sigma^{2}}$ between the $i-t h$ transmit and $j-t h$ receive angle pair for $i=1, \ldots, P ; j=1, \ldots, Q$.

1) High SNR Case: Consider the high RSNR case, where $\operatorname{RSNR}(\mathrm{i}, \mathrm{j}) \gg 1$. Let $Q_{i}$ be the number of active elements in the $i$-th column (or equivalently the number of active receive elements the $i$-th transmit beam couples with). Using Langrange multipliers, it can be shown that for high RSNR case, MMSE and CMI criteria assign power according to

$$
\begin{aligned}
& \text { MMSE : } \quad \beta_{i}=\frac{\sqrt{Q_{i}} \beta}{\sum_{i=1}^{P} \sqrt{Q_{i}}} \quad i=1, \ldots, P, \\
& \text { and CMI : } \quad \beta_{i}=\frac{Q_{i} \beta}{\sum_{i=1}^{P} Q_{i}} \quad i=1, \ldots, P,
\end{aligned}
$$

respectively. Thus, the CMI (MMSE) criterion assigns power to the transmit beams in proportion to the sum (square root of the sum) of the active elements they couple with at the receiver.
Remark: In the extreme case, when all the elements of $\mathbf{H}_{V}$ are active, then equal power is distributed at all transmit branches for both the criteria.

2) Low SNR Case: Consider the low RSNR case, where $\operatorname{RSNR}(\mathrm{i}, \mathrm{j}) \ll 1, \quad \forall \mathrm{i}, \mathrm{j}$. Using Langrange multipliers, it can be shown that the MMSE and CMI criteria assign all the power $\beta$ to the $k$-th transmit beam such that

$\operatorname{MMSE}: \quad k=\arg \max _{i} \sum_{j=1}^{Q} \mathbf{R}_{V}^{2}((i-1) Q+j,(i-1) Q+j)$,

CMI : $\quad k=\arg \max _{i} \sum_{j=1}^{Q} \mathbf{R}_{V}((i-1) Q+j,(i-1) Q+j)$,

respectively. Thus at low RSNR, the CMI (MMSE) criterion assigns all the power to that transmit angle for which, the sum (sum of squares) of the variances of the corresponding virtual receive elements is maximum.

From the extreme cases, we conclude that the number of transmit beams to be sent and hence the block length $K$ depends on the SNR. For high SNR, $K$ is determined by the number of active columns (which can be determined a priori from $\mathbf{R}_{V}$ ) and have a maximum value of $K=P$. Whereas for low SNR, $K=1$ and all the power is transmitted in the strongest transmit direction. For medium SNR, $1 \leq K \leq P$ and the powers will be determined by the water filling criteria. Also note that for i.i.d. channels, equal power will be assigned to all transmit beams irrespective of the SNR.

\section{INTERPRETATION AND SIMULATIONS}

The optimal signal is a block of length $K \leq P$ and has a diagonal structure given by $\mathbf{S}_{V}=\Lambda_{S}$. The block $\mathbf{S}_{V}$ represents beams transmitted in succession along the fixed virtual transmit angles, with the powers given by the water filling arguments (18) and (19) for the MMSE and CMI criteria, respectively. Basically, the scattering environment is scanned along the virtual transmit angles, one by one, and the presence of scatterers is determined, by measuring the signal along the receive virtual angles for each transmitted beam. In other words, the $i$-th transmitted beam is used to estimate the $i$-th column of $\mathbf{H}_{V}$. Depending on (the diagonal entries of) $\mathbf{R}_{V}$ and the SNR, power is assigned to the beams by water filling, which identifies the set of virtual transmit angles that couple strongly enough with receive angles or effectively the dominant subspaces of the channel. Hence the block length $K$, which is exactly equal to the size of this set, depends on the SNR and $\mathbf{R}_{V}$. In particular, for low SNR, $K=1$, while for high SNR $K$ has a maximum value equal to the number of active columns determined from $\mathbf{R}_{V}$ (which has a maximum of $P$ ) and for medium SNR, $1 \leq K \leq P$. This in turn implies that at low SNR, a faster fading rate can be tolerated than at high SNR, since fewer essential parameters need to be estimated. For high SNR, the CMI (MMSE) criterion assigns the power to the transmit angles in proportion to the sum $(\sqrt{\mathrm{sum}})$ of the active elements they couple with at the receiver. As the SNR decreases further, the weakest transmit beam (as determined by the water filling 
criteria) is dropped. As the SNR decreases, this process continues until finally the CMI (MMSE) criterion assigns all the power to the strongest transmit beam, that is one for which the sum (sum of squares) of the variances of the corresponding virtual receive elements is maximum.

These interpretations of our analytical results are confirmed by the numerical results presented in Figures 2 - 5. In all the figures, the total transmit power (in $\mathrm{dB}$ ) along the $x$-axis is given by $10 \log _{10}\left(\beta / \sigma^{2}\right)$, while the $y$-axis shows the branch powers in $\mathrm{dB}$ given by $10 \log _{10}\left(\beta_{i} / \sigma^{2}\right)$. Powers are plotted for for both MMSE and CMI criteria and the equal power assignment is also plotted for comparison. Figure 2 shows the power assignments for the MISO case with $P=2$ where the covariance matrix is given by $\mathbf{R}_{V}=\operatorname{diag}\left(\left[\begin{array}{ll}1 & 0.05\end{array}\right]\right)$ or equivalently for a MIMO case with $P=Q=2$ and $\mathbf{R}_{V}^{(1)}=\operatorname{diag}\left(\left[\begin{array}{llll}1 & 0 & 0 & 0.05\end{array}\right]\right)$ where the first two components are the variances of the elements in the first column of $\mathbf{H}_{V}$ and the next two are those of the second column. The number of active elements in both the columns is one, i.e $Q_{1}=Q_{2}=1$. Both criteria give identical power assignment in this case [8]. Observe that the second transmit beam gets non-zero power only after the SNR increases to about $13 \mathrm{~dB}$ (this behavior is similar in the next three figures too). For high SNR, however, both transmit beams get equal power. The following three figures are also for the MIMO case with $P=Q=2$. In Figure 3, we make the variance of the first element of the second column of $\mathbf{H}_{V}$ nonzero to get $\mathbf{R}_{V}^{(2)}=\operatorname{diag}\left(\left[\begin{array}{llll}1 & 0 & 0.01 & 0.05\end{array}\right]\right)$, so that the number of active elements in the two columns of $\mathbf{H}_{V}$ are $Q_{1}=1$ and $Q_{2}=2$. Observe that for high SNR, the second transmit beam gets $66 \%$ (58\%) power according to CMI (MMSE) criterion. The powers are reversed in Figure 4 where $\mathbf{R}_{V}^{(3)}=\operatorname{diag}\left(\left[\begin{array}{llll}1 & 0.1 & 0 & 0.05\end{array}\right]\right)$, so that now we have $Q_{1}=2$ and $Q_{2}=1$. Finally in Figure 5 we have $\mathbf{R}_{V}^{(4)}=\operatorname{diag}\left(\left[\begin{array}{llll}1 & 0.1 & 0.01 & 0.05\end{array}\right]\right)$ and $Q_{1}=Q_{2}=2$. Note that at high SNR both branches get equal power as in Figure 2. However, the power assignment in the medium SNR range is different in the two figures. In all cases, as the SNR decreases the weaker beam is dropped and the stronger beam gets all the power.

\section{REFERENCES}

[1] D. Shiu, G. Foschini, M. Gans, and J. Kahn, "Fading correlation and its effect on the capacity of multielement antenna systems," IEEE Transactions on Communications, vol. 48, no. 3, pp. 502-512, Mar. 2000.

[2] E. Telatar, "Capacity of multi-antenna Gaussian channels," AT \& T Bell Labs Internal Technical Memo, June 1995.

[3] G. J. Foschini, "Layered space-time architechture for wireless communication in a fading environment when using multi-element antennas," Bell Labs Technical Journal, vol. 1, no. 2, pp. 41-59, 1996.

[4] A. J. Paulraj and C. B. Papadias, "Space-time processing for wireless communications," IEEE Signal Proc. Magazine, pp. 49-83, Nov. 1997.

[5] A. M. Sayeed, "Deconstructing multi-antenna fading channels," IEEE Transactions of Signal Processing, Oct. 2002.

[6] S. A. Jafar and A. Goldsmith, "Multiple-antenna capacity in correlated Rayleigh fading with no side information," preprint: http://wsl.stanford.edu/Publications.html, pp. 2266-2270, 2001.

[7] J. W. Brewer, "Kronecker products and matrix calculus in system theory," IEEE Trans. on Circuits and Systems, vol. 25, pp. 772-781, Sep. 1978.

[8] J. H. Kotecha and A. M. Sayeed, "Transmit signal design for optimal estimation of correlated MIMO channels," Submitted to IEEE Transactions on Signal Processing, special issue on MIMO, Dec 2002.

[9] B. Hassibi and B. Hochwald, "Optimal training in space-time systems," Conference Record of the Thirty-Fourth Asilomar Conference on Signals, Systems and Computers, vol. 1, pp. 743 -747, 2000.

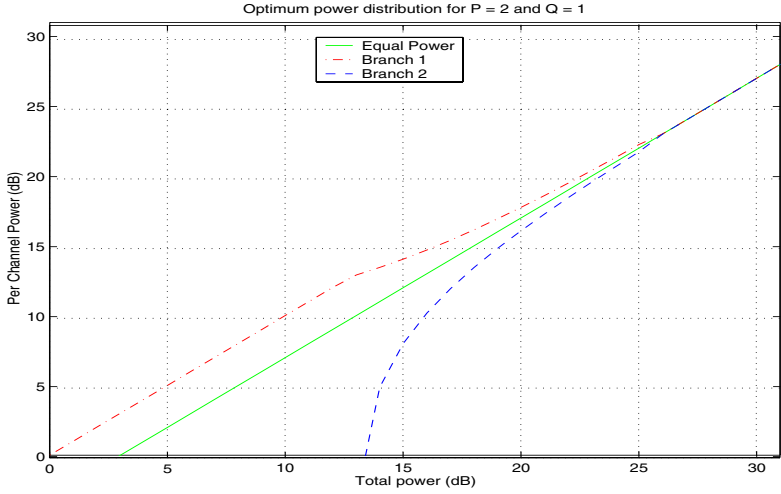

Fig. 2. Optimal power distribution for $P=2$ and $Q=1$.

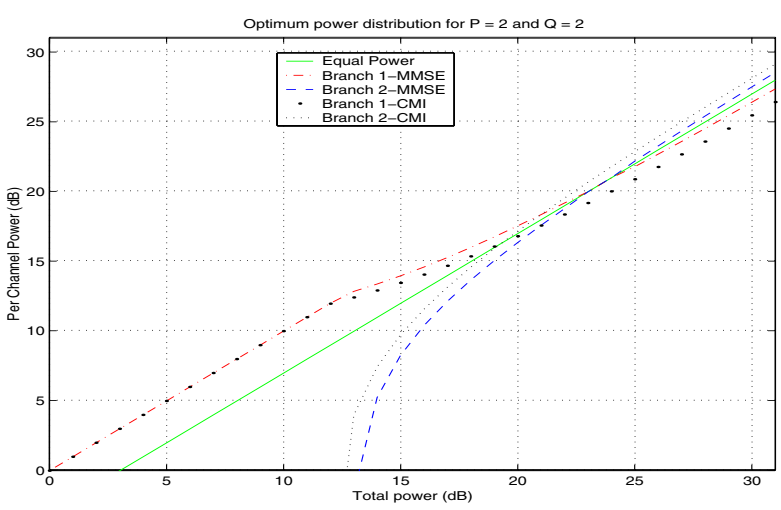

Fig. 3. Optimal power distribution for $P=2$ and $Q=2$ and $\mathbf{R}_{V}=\mathbf{R}_{V}^{(2)}$.

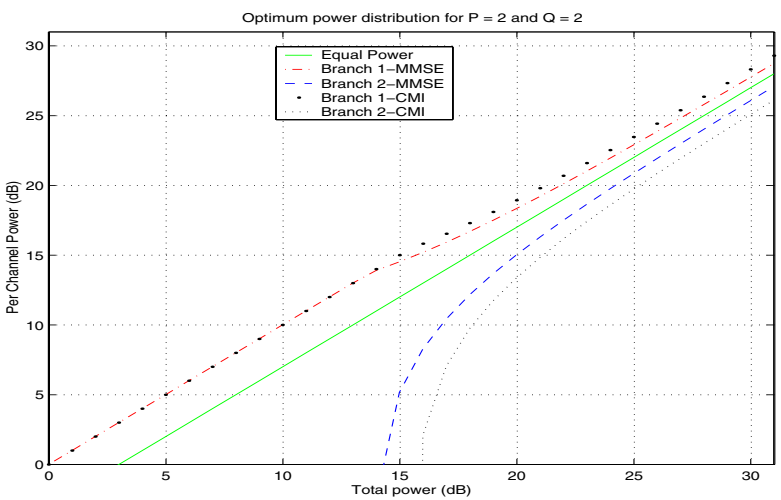

Fig. 4. Optimal power distribution for $P=2$ and $Q=2$ and $\mathbf{R}_{V}=\mathbf{R}_{V}^{(3)}$.

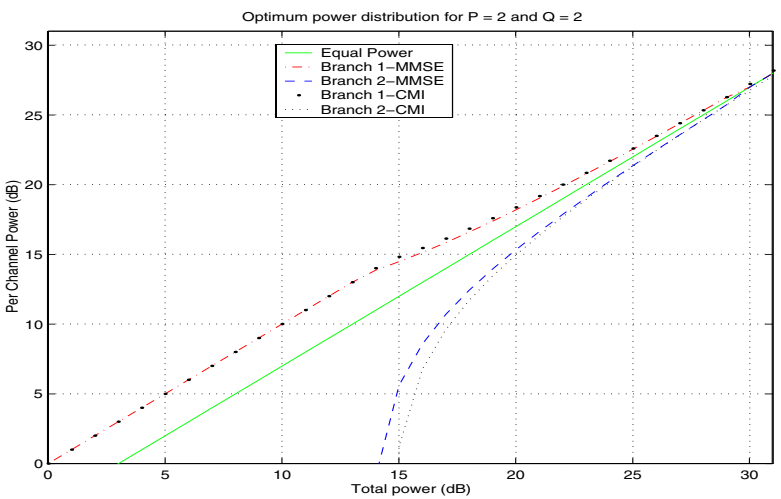

Fig. 5. Optimal power distribution for $P=2$ and $Q=2$ and $\mathbf{R}_{V}=\mathbf{R}_{V}^{(4)}$. 